\title{
Correlation of amplification and overexpression of the c-myc oncogene in high-grade breast cancer: FISH, in situ hybridisation and immunohistochemical analyses
}

\author{
J Blancato*,1,2, B Singh ${ }^{2}$, A Liư ${ }^{3}$, DJ Liao ${ }^{4}$ and RB Dickson ${ }^{2,5}$ \\ 'Institute for Molecular and Human Genetics, 3970 Reservoir Road, NW, Washington DC 20007, USA; '2 Lombardi Comprehensive Cancer Center, 3970 \\ Reservoir Road, NW, Washington DC 20007, USA; ${ }^{3}$ Biometry and Mathematical Statistics Branch, National Institute of Child Health and Human \\ Development, 9000 Rockville Pike, Bethesda, MD 20892-75 I0, USA; ${ }^{4}$ Department of Radiation Oncology, Karmanos Cancer Institute, Wayne State \\ University, Detroit, MI, USA; ${ }^{5}$ Department of Oncology, Georgetown University Medical Center, 3970 Reservoir Road, NW, Washington DC 20007, USA
}

In this study, we analysed gene amplification, RNA expression and protein expression of the c-myc gene on archival tissue specimens of high-grade human breast cancer, using fluorescent in situ hybridisation (FISH), nonradioactive in situ hybridisation and immunohistochemistry. The specific question that we addressed was whether expression of c-Myc mRNA and protein were correlated with its gene copy amplification, as determined by FISH. Although c-Myc is one of the most commonly amplified oncogenes in human breast cancer, few studies have utilised in situ approaches to directly analyse the gene copy amplification, RNA transcription and protein expression on human breast tumour tissue sections. We now report that by using the sensitive FISH technique, a high proportion (70\%) of high-grade breast carcinoma were amplified for the c-myc gene, irrespective of status of the oestrogen receptor. However, the level of amplification was low, ranging between one and four copies of gene gains, and the majority (84\%) of the cases with this gene amplification gained only one to two copies. Approximately $92 \%$ of the cases were positive for cmyc RNA transcription, and essentially all demonstrated c-myc protein expression. In fact, a wide range of expression levels were detected. Statistically significant correlations were identified among the gene amplification indices, the RNA expression scores and protein expression scores. c-myc gene amplification, as detected by FISH, was significantly associated with expression of its mRNA, as measured by the intensity of in situ hybridisation in invasive cells $(P=0.0067)$, and by the percentage of invasive cells positive for mRNA expression $(P=0.0006)$. c-myc gene amplification was also correlated with the percentage of tumour cells which expressed high levels of its protein, as detected by immunohistochemistry in invasive cells $(P=0.0016)$. Thus, although multiple mechanisms are known to regulate normal and aberrent expression of c-myc, in this study, where in situ methodologies were used to evaluate highgrade human breast cancers, gene amplification of c-myc appears to play a key role in regulating expression of its mRNA and protein British Journal of Cancer (2004) 90, 1612-1619. doi:10.1038/s.bjc.6601703 www.bjcancer.com

Published online 30 March 2004

(c) 2004 Cancer Research UK

Keywords: c-myc; breast cancer, gene amplification; gene expression

The c-myc oncogene has been shown to be amplified and/or overexpressed in many types of human cancer (Marcu et al, 1992; Nass and Dickson, 1997; Nesbit et al, 1999; Liao and Dickson, 2000). Numerous experiments in vivo have also causally linked aberrant expression of this gene to the development and progression of cancer in different body sites (Marcu et al, 1992; Nass and Dickson, 1997; Nesbit et al, 1999; Liao and Dickson, 2000). However, several critical issues regarding the significance of $c-m y c$ in human cancer still remain obscure. First, even for a given type of malignancy, the frequencies of the alterations of c-myc at the cytogenetic and expression levels vary greatly from one report to another (Liao and Dickson, 2000). For instance, the frequencies of its amplification, mRNA and protein overexpression in breast cancer vary between 1-94, 22-95 and roughly 50-100\%, respectively, among different reports (Liao and Dickson, 2000).

*Correspondence: Dr J Blancato; E-mail: blancatj@georgetown.edu Received 7 July 2003; revised 17 January 2004; accepted 17 January 2004; published online 30 March 2004
Thus, it is still unclear to what extent this gene is altered at the cytogenetic level and at different expression levels in breast carcinoma.

One controversial issue pertains to the prognostic value of $\mathrm{c}-m y c$ gene alterations in cancer. The central role of $c-M y c$ protein in accelerating cell proliferation, documented by many early studies, has led to a general concept for many types of cancer that amplification or overexpression of this gene may be associated with a more aggressive tumour and a poorer patient survival (Berns et al, 1992; Marcu et al, 1992; Sato et al, 1995; Nass and Dickson, 1997; Nesbit et al, 1999; Visca et al, 1999; Liao and Dickson, 2000). However, many reports have shown an opposite correlation (Sikora et al, 1985, 1987; Watson et al, 1986; Polacarz et al, 1989; Voravud et al, 1989; Williams et al, 1990; Melhem et al, 1992; Pietilainen et al, 1995; Diebold et al, 1996; Smith and Goh, 1996; Augenlich et al, 1997; Bieche et al, 1999), while other studies do not support either of these conclusions. For instance, gene amplification or overexpression of c-Myc protein has also been shown to associate with a better tumour differentiation or a better 
patient survival for cancer of the testis, ovary, bile ducts, colon and breast (Sikora et al, 1985, 1987; Watson et al, 1986; Polacarz et al, 1989; Voravud et al, 1989; Williams et al, 1990; Melhem et al, 1992; Pietilainen et al, 1995; Diebold et al, 1996; Smith and Goh, 1996; Augenlich et al, 1997; Bieche et al, 1999). This controversy does not appear to be related completely to the cancer type, since both positive (Berns et al, 1992; Visca et al, 1999) and negative (Williams et al, 1990; Melhem et al, 1992; Pietilainen et al, 1995; Smith and Goh, 1996; Augenlich et al, 1997; Bieche et al, 1999) correlations have been reported for colon cancer and breast cancer. More interestingly, c-Myc overexpression has been shown to predict a poorer prognosis for cutaneous melanoma, but a favourable outcome for uveal melanoma (Grover et al, 1997; Chana et al, 1998a, b, 1999; Grover et al, 1999). These data indicate different roles of c-Myc, even in the same type of tumour, perhaps depending upon different tissue microenvironments.

Another controversial issue concerns the nuclear-cytoplasmic localisation of c-Myc. Studies of neoplasms of the colon, testis, ovary and liver have shown that predominantly nuclear localisation of c-Myc tends to occur in benign lesions, while cytoplasmic localisation tends to occur in more malignant tumours (Sikora et al, 1985; Sundaresan et al, 1987; Melhem et al, 1992; Sasano et al, 1992; Yuen et al, 2001). Whether these patterns of subcellular localisation of c-Myc tend to reflect the malignant status of breast cancer remains an enigma.

A recent study of the impact of DNA amplification on gene expression patterns in breast cancer used mRNA and DNA from 14 breast cancer cell lines. Analysis was conducted with a 13000 cDNA clone array for gene expression measurement and a Comparative Genomic Hybridisation (CGH) microarray for gene copy number measurements. This study also included known breast cancer genes, such as c-myc, HER/2-neu and aib1 (Hyman et al, 2002). Interestingly, $44 \%$ of the most highly amplified genes were also overexpressed at the mRNA level. Consistent with this pattern, c- $M y c$ gene copy number and its expression levels showed a statistically significant $(\alpha=0.020)$ correlation in this microarray study of breast cancer cell lines. Another study, by Pollack and colleagues, used microarray analysis and BAC array CGH of RNA and DNA (respectively) extracted from intermediate grade human breast tissues, and tested for amplification and expression of cMyc (among other genes). This study demonstrated that two out of 37 specimens were both amplified and overexpressed, while others were either amplified or overexpressed, but not both. The authors of this study suggested that contaminating stromal tissue may compress the fluorescence ratios leading to underestimates of gene amplification and overexpression (Pollack et al, 2002).

To more clearly address the importance of gene amplification and expression of c-Myc in human breast cancer, we used in situ methodologies, which can clearly distinguish stromal and carcinoma components. We studied the amplification and over expression of the c-myc gene with fluorescent in situ hybridisation (FISH), non-radioactive in situ hybridisation (ISH) and immunohistochemical (IHC) approaches on paraffin-embedded biopsy sections of untreated, high-grade breast cancer. It was observed that 70,92 and $70 \%$ of the cancer cases exhibited c-myc gene amplification, its mRNA overexpression and its protein over expression, respectively. In most of the cases (84\%) that showed gene amplification, the $c-m y c$ gene gained only one to two copies, which is consistent with c-myc FISH data from other studies. Unlike some oncogenes, such as N-myc, which typically demonstrates gene amplification copy numbers of greater then 10 in neuroblastoma, and HER-2/neu (Sartelet et al, 2002), whose copy numbers range up to $14-40$ in breast carcinomas (Isola et al, 1999), gene copy numbers of c-myc are not as greatly increased. In the study noted earlier, using breast cancer cell line CGH array and cDNA microarray expression analysis, it was demonstrated that the most dramatically increased expression levels were associated with large gene copy number increases, although low-level gains and losses had a significant influence on gene expression dysregulation (Hyman et al, 2002). Only one study has been published (Pollack et al, 2002) that has begun to determine if these findings are directly relevant to actual human breast tumour tissues, since many of the genetic changes in tissue culture cell lines are more extreme than those displayed in primary tumour material. Furthermore, the relationships among gene amplification, mRNA expression and c-Myc protein expression were not explored in prior human breast cancer cell line and tumour tissue studies (Hyman et al, 2002; Pollack et al, 2002).

In our human breast tumour tissue study, a high correlation was found between $c-m y c$ FISH and ISH, for both percentage of staining $(P<0.0067)$ and intensity positive cells $(P<0.0006)$. In addition, c-myc gene copy amplification by FISH was correlated with $\mathrm{c}-\mathrm{Myc}$ protein expression positive cells by IHC $(P<0.0016)$. These results support the idea that c-Myc overexpression of both mRNA and protein is related to the copy number of the $c-m y c$ DNA amplification. We show in this study that amplification and overexpression of c-Myc occur with high frequency in high-grade human breast cancer tissues.

\section{MATERIALS AND METHODS}

\section{Materials}

Formalin-fixed, paraffin-embedded tissue blocks of breast carcinoma and normal breast tissue were obtained from the Histopathology and Tissue Shared Resource at the Lombardi Comprehensive Cancer Center (LCCC), at Georgetown University Medical Center. The criteria for tumour selection were the following: negative progesterone receptor status, metastases to auxiliary lymph nodes and high grade (Elston Score $>7$ ). The oestrogen receptor status of the tumours was known from archived pathology reports. The parameters were chosen from our prior meta-analysis (Deming et al, 2000), as indications of a high likelihood of c-myc gene amplification. Normal breast tissue specimens were from reduction mammoplasty. Serial sections $(5 \mu \mathrm{m})$ for FISH, ISH and IHC were prepared by the LCCC Histopathology and Tissue Shared Resource.

\section{FISH}

A dual-label FISH technique was used (Jenkins et al, 1997). Slides were baked overnight at $60^{\circ} \mathrm{C}$ to assure adherence of the sample. Tissue sections were deparaffinised with two successive, $10 \mathrm{~min}$ xylene washes, and then dehydrated in a graded ethanol series of 70,80 and $95 \%$ at room temperature. Samples were then digested with $4 \%$ pepsin (Sigma, St Louis, MO, USA) at $45^{\circ} \mathrm{C}$ for $10 \mathrm{~min}$. DNA probes used were an alpha satellite probe to chromosome 8 , labelled with biotin, and a $c-m y c$ probe, labelled with digoxigenin (Ventana, Tucson, AZ, USA). Codenaturation was performed at $90^{\circ}$ for $10 \mathrm{~min}$ on a hot plate. Hybridisation was at $37^{\circ} \mathrm{C}$ for $12-$ $16 \mathrm{~h}$. Detection of signals was accomplished with an antiavidin antibody labelled with Texas Red, and an antidigoxigenin antibody conjugated to fluorescein (Ventana, Tucson, AZ, USA). Slides were postwashed in $2 \times \mathrm{SSC}$ at $72^{\circ} \mathrm{C}$ for $5 \mathrm{~min}$ and counterstained with DAPI to visualise cell nuclei. Results were viewed and quantified with a Zeiss Axiophot fluorescence microscope, equipped with appropriate filters and an Applied Imaging Cytovision system (Pittsburgh, PA, USA). In this approach, the c-myc unique sequence probe was visualised as a green signal and the control probe for the chromosome 8 centromere was red, thus easily being distinguished when scored.

One serial section from each tumour sample was stained with haematoxylin and eosin and first reviewed by a pathologist (BS), to help identify the tumour area of the section. This procedure ensured that the tumour cells, but not the normal cells, were 
counted. Nuclei of up to 50 tumour cells were scored from each FISH-stained section, independently by two investigators. Hybridisation signals were averaged, and the amplification index was presented as the number of c-myc signals divided by the number of chromosome 8 centromere signals. A 1.8-fold increase was used as the criterion to judge the presence of $c-m y c$ gene amplification.

\section{In situ hybridisation}

In situ hybridisation (ISH) was carried out with a nonradioactive method, described previously (Liao et al, 2000a, b). One serial section from each specimen was hybridised overnight at $60^{\circ} \mathrm{C}$ with riboprobes, that were in vitro transcribed from the antisense or sense strand of an approximately $300 \mathrm{bp} c \mathrm{cNA}$ of human c-myc (ATCC, Manassas, VA, USA), labelled with digoxigenin-conjugated UTP. The sections were then incubated with an antibody against digoxigenin, followed by incubation with a second antibody conjugated to alkaline phosphatase. The signal was visualised by colour development with 5 bromo-4-chloro-3-indolyl phosphate and nitroblue tetrazolium. All reagents were purchased from Boehringer Mannheim, Indianapolis, IA. To control the signal specificity, two serial sections were mounted on the same slide for hybridisation with the antisense and sense probes, respectively. ISH was given an intensity and percentage scores, based on intensity of positive staining and number of cells staining, respectively. Intensity scores were assigned $0,1,2$ and 3 , and percentage scores were assigned as 1-1-25, 2- 26-50, 3- 51-75 and $4-76-100 \%$.

\section{Immunohistochemistry}

Immunohistochemical staining (IHC) was performed using an avidin-biotin complex (ABC) method described previously (Liao et al, 1998). One serial section of each specimen was deparaffinised and blocked with $3 \%$ peroxide. Antigens were retrieved by heating slides in a microwave oven in $50 \mathrm{~mm}$ citrate buffer, $\mathrm{pH} 6.4$, at boiling temperature, for $12 \mathrm{~min}$. After blocking with $6 \%$ normal goat serum, the section was incubated with a mouse monoclonal antibody to human c-Myc (9E10, Sigma Chemical Company, St Louis, MO, USA) at 1:100 dilution for $2 \mathrm{~h}$, followed by $1 \mathrm{~h}$ incubation with a second antibody conjugated with biotin (Vector Laboratories Inc., Burlingame, CA, USA). The section was then incubated with peroxidase-conjugated avidin (Dako, Corporation, Carpinteria, CA, USA) for $30 \mathrm{~min}$, followed by colour development with diaminobenzidine and peroxide. All procedures were carried out at room temperature. To control the signal specificity, serial sections from 10 tumour samples were also stained using an alternate c-Myc antibody (C19 from Santa Cruz Biotechnology Inc., Santa Cruz, CA, USA) at 1:60 dilution. This antibody resulted in focally positive staining in the tumour, but the staining intensity was weaker. To control the signal specificity, serial sections were made from five selected positive cases which were subjected to the same staining procedure, with a normal mouse IgG to replace the c-Myc antibody. This control staining did not give rise to a signal, demonstrating the specificity of the c-Myc antibody signal. IHC staining was given an intensity and percentage score based upon the intensity of positive staining and number of cells staining. Intensity scores were assigned $0,1,2$ and 3 and percentage scores were assigned as 1- 1-25, 2- 26-50, 3- 51-75 and 4- 76-100\%. Determinations were made of cellular localisation of c-Myc antibody staining to cytoplasm and/or nucleus in normal and invasive cells within each breast tumour specimen.

\section{Statistical analyses}

For each analysis of gene copy amplification (FISH), mRNA expression (ISH) and protein expression (IHC), all cases were first grouped as positive or negative to calculate the percentages of positive cases and negative cases, as described (Zar, 1974). Fisher's exact test was used to compare percentages, and two-sample $t$-test or Wilcoxon rank test was used to compare average scores. Both ISH and IHC were given intensity and percentage scores, based on intensity of positive staining and number of cells staining, respectively. As noted earlier, intensity scores were assigned 0,1 , 2 and 3 and percentage scores were assigned as 1- 1-25, 2- 26-50, 3- 51-75 and 4- 76-100\%. A score of $>2$ for either intensity of staining or percentage of cells positive by ISH was assigned as high. For IHC, an intensity score of $>1$ was assigned as high and a percentage score of $>3$ was categorised as high. Each amplification index was paired with its corresponding mRNA expression score to calculate the coefficient $r$. The same method was used to estimate the association of the amplification indices with the cMyc protein expression levels, and the association of the mRNA expression levels with the protein expression levels. A $P$-value of 0.05 or less was used to determine the statistical significance in all analyses. In all, 54 pairs of normal $v s$ invasive tissues were analysed using McNemars $\chi^{2}$ test to determine if there was a difference in cellular localisation of c-Myc antibody signal to nuclear or cytoplasmic compartments.

\section{RESULTS}

\section{FISH analysis of gene amplification}

Amplification of the c-myc gene was measured by a FISH test in 46 cases of breast cancer; Figure 1 demonstrates cells with no amplification (one copy of c-myc /one copy of chromosome 8 centromere, and a moderate amplification a $3 / 1$ ratio). Amplification was calculated by the number of $c-m y c$ signals divided by the number of chromosome 8 alpha satellite signals. A 1.8-fold increase cut-off was used to judge gene amplification. As shown in Table 1, 32 out of $46(70 \%)$ cases were gene amplified for c-myc, whereas only $30 \%(14 / 46)$ of the cases showed amplification indices lower than the cut-off value. The amplification indices for most $(84 \%$, or $27 / 32)$ cases with gene amplification, ranged between 1.8- and three-fold, indicating that the locus gained up to two copies of $c-m y c$ in the majority of the cases. The percentage of cases with gene gains of three copies or higher was $11 \%$ (five out of 46) of total cases analysed, or near $16 \%$ (five out of 32) of the cases with gene amplification, including one case $(2 \%$ of total cases or $3 \%$ of the cases with gene amplification) with the highest index of 5 (a gain of four copies).

In all, 28 of the breast carcinomas in this study were ER negative, and 14 were ER positive. The average c-myc gene amplification score was 1.896 (s.e. $=0.196)$ for ER positive and 2.201 (s.e. $=0.157$ ) for ER negative. Although ER-negative tumours had a slightly higher average c-myc score, the difference was not statistically significant (two-sided $P=0.252$ from two-sample $t$-test and 0.251 from Wilcoxon rank test), consistent with the results of our prior meta-analysis of the literature (Deming et al, 2000).

\section{In situ hybridisation analysis of c-myc mRNA expression}

A total of 51 breast cancer samples were studied for c-Myc mRNA expression, with non radioactive in situ hybridisation (ISH). ISH results were assigned intensity and percentage scores based upon signal intensity of positive staining and number of cells staining within the sample, respectively. As shown in Table 2, 86\% (44 out of 51) tumours were scored as high in intensity, and $92 \%$ (47out of 51) had more than $51 \%$ positive cells, also considered as highly increased c-Myc expression. mRNA expression was heterogeneous in the breast tumour tissue, and no morphologic subtype was predominant in the high or low categories. One case showed no cMyc ISH staining. In 79\% (38/48) of cases, epithelia in normal mammary glands adjacent to the tumour also showed a high 
A

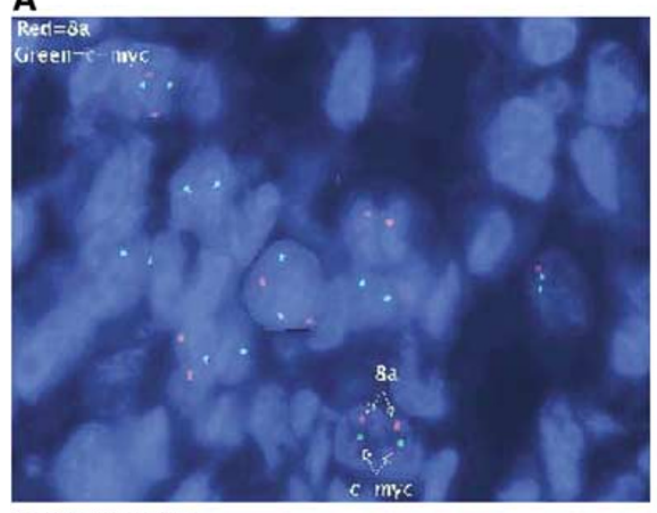

Normal results
B

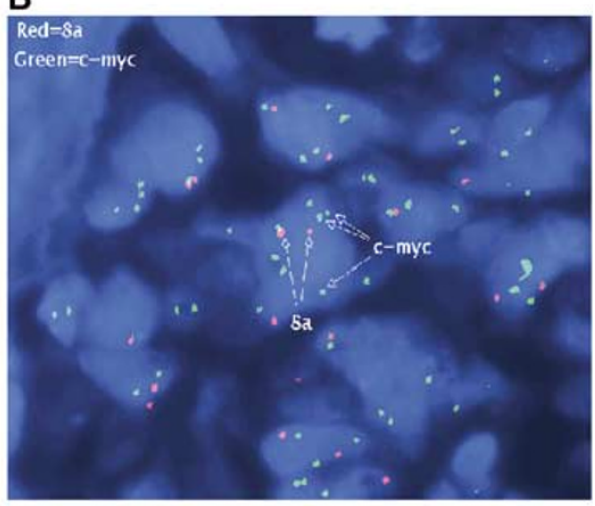

c-myc Amplification

Figure I FISH analysis of c-myc amplification in tumour cells from breast tumour tissue sections. FISH probe for human c-myc unique-sequence is seen as green, while the normal control signal, a centromeric probe signal for chromosome 8 is shown in red. The nuclei of tumour cells were visualised by DAPI counter-staining. (A) I : I copy ratio of c-myc to chromosome 8 (c-myc/8 centromere), indicating no amplification of c-myc in tumour cells. (B) I : 3 copy ratio of c-myc to chromosome 8 (c-myc/8 centromere), a moderate amplification of the c-myc gene.

Table I c-myc gene copy amplification analysis by FISH in poor prognosis human breast tumour samples

\begin{tabular}{lll}
\hline $\begin{array}{l}\text { Amplification index } \\
\text { (\#c-myc signals/\# } \\
\text { control signals)+ }\end{array}$ & $\begin{array}{l}\text { Percentage of samples with } \\
\text { FISH ratios in each category }\end{array}$ \\
\hline $1.0-1.7$ & $30 \%$ & 14 out of 46 \\
$1.8-1.99$ & $20 \%$ & Nine out of 46 \\
$2.0-2.9$ & $39 \%$ & 18 out of 46 \\
$>3.0$ & $11 \%$ & Five out of 46 \\
\hline
\end{tabular}

Analysis was conducted on 46 individual paraffin-embedded tissue samples with negative progesterone receptor status, positive lymph node involvement and high tumour grade. +Normal control ratio is 1 .

intensity of staining. In three cases, no staining was seen in the normal terminal duct lobular units. Figure 2 shows representative fields of high, medium and low $c$-myc mRNA expression levels in invasive ductal carcinoma samples.

\section{Association of FISH and ISH}

c-Myc scores were dichotomised as binary variables (high or low), and a score of 2 or higher was categorised as high on ISH. A score higher than median was categorised as high from FISH studies. These dichotomised scores are depicted in Table 3. A Fisher's exact test was performed for comparing binary responses to see if there was any association between FISH and ISH. It was found that the FISH score was significantly associated with percentage of staining in the invasive cells ( $P=0.0067$, two-sided McNemar's test) and also with the intensity score on ISH ( $P=0.0006$, two-sided).

\section{Immunohistochemical staining of c-Myc proteins}

In total, 51 breast carcinomas, which were subjected to FISH analysis, and all of which also had been analysed for c-myc mRNA by in situ hybridisation, were also analysed for the expression of cMyc protein, using immunohistochemical staining with the 9E10 antibody. IHC results were assigned an intensity and percentage score based on intensity of positive staining and number of cells staining, respectively. Intensity scores were assigned $0,1,2$ and 3 and percentage scores were assigned as $0,1-0-25,2-26-50,3-$
51-75 and 4- 76-100. For IHC, an intensity score of $>1$ was assigned as high and a percentage score of $>3$ was categorised as high. Figure 2 shows examples of high, medium and low levels of cmyc antibody staining in invasive ductal carcinoma samples. In 34 cases, normal tissue was seen; 30 of these showed cytoplasmic staining and 22 had nuclear staining in terminal ductal lobular units. In all, 12 cases showed $1+, 14$ cases $2+$ and four cases $3+$ cytoplasmic staining. In situ hybridisation revealed positive staining in 46 out of 49 cases with normal tissue. Seven cases showed $1+, 13$ cases showed $2+$ and 26 cases showed $3+$ staining by ISH. Both immunohistochemistry and in situ hybridisation showed diffuse positivity in adipocytes.

Table 4 shows the staining pattern for the cohort. In all, 70\% (36 out of 51) of cases showed high intensity of staining for c-Myc protein, while $85 \%$ ( 29 out of 34 ) of cases with detectable staining had more than $76 \%$ positive cells, also considered as high expression. To verify the staining specificity, serial sections from 10 tumour specimens that were positive for 9E10 antibody were also stained using the $\mathrm{C} 19$ rabbit polyclonal anti-c-Myc antibody. Results revealed a staining pattern similar to 9E10. However, the staining intensity with C19 was weaker than 9E10. The specificity of these two antibodies was verified by Western blots in previous studies (Persons et al, 1997; Liao et al, 2000b). Figure 2 shows results of c-Myc in situ hybridisation and immunohistochemistry studies on samples considered to demonstrate low, moderate and high levels of c-Myc expression. Analysis of c-Myc protein localisation results in the nucleus or cytoplasmic compartments of normal and invasive cells within the tumours revealed that nuclear staining was positive in $41 \%$ of normal cells, compared to $22 \%$ of invasive cells (statistical significance at $P=0.01$ by McNemar's two-sided $\chi^{2}$ test). The increase in relative cytoplasmic localisation of c-Myc protein, comparing normal (53.7\%), to invasive cells $(61.1 \%)$ was not significantly different. Thus, the data are consistent with partial exclusion of $\mathrm{c}-\mathrm{Myc}$ from the nuclei of invasive breast cancer cells.

The FISH score was significantly associated with the percentage positivity of invasive cells, as seen on IHC studies of c-Myc. However, $40 \%$ of tumours displayed a low index of c-myc gene amplification, but still expressed high levels of c-Myc protein (Table 6), indicating the possibility of other mechanisms of over expression unrelated to gene amplification in at least some tumours. The FISH score was not significantly associated with the intensity of IHC staining in the invasive cells (not shown), in contrast to the IHC percentage positivity score. 
Table 2 c-myc mRNA in situ hybridisation (ISH) results

\begin{tabular}{lrrrrrrrrr}
\hline Staining intensity & $\mathbf{0}$ & $\mathbf{l}$ & $\mathbf{2}$ & $\mathbf{3}$ & Percent positivity & $\mathbf{3}$ & $\mathbf{4}$ \\
\hline Number of tumour samples in each category $N=51$ & 1 & 6 & 25 & 19 & Number of tumour samples in each level category $N=51$ & 1 & 3 & 42
\end{tabular}

In all, 5 I human high-grade breast carcinomas were analysed to determine the relationships between c-Myc mRNA expression and c-myc gene in situ hybridisation results. Data are shown in two ways in the above table. First, overall staining intensity of c-Myc-positive cells was scored as 0, I, 2, 3 (low to high), and the number of tumour samples at each level of staining indicated on the line below. Next, the percentage of tumour cells staining was scored as 0, I, 2, 3, 4 (low to high \%, as discussed in Materials and Methods). The number of tumours at each level of percent cell positivity for c-Myc is then indicated on the line below.
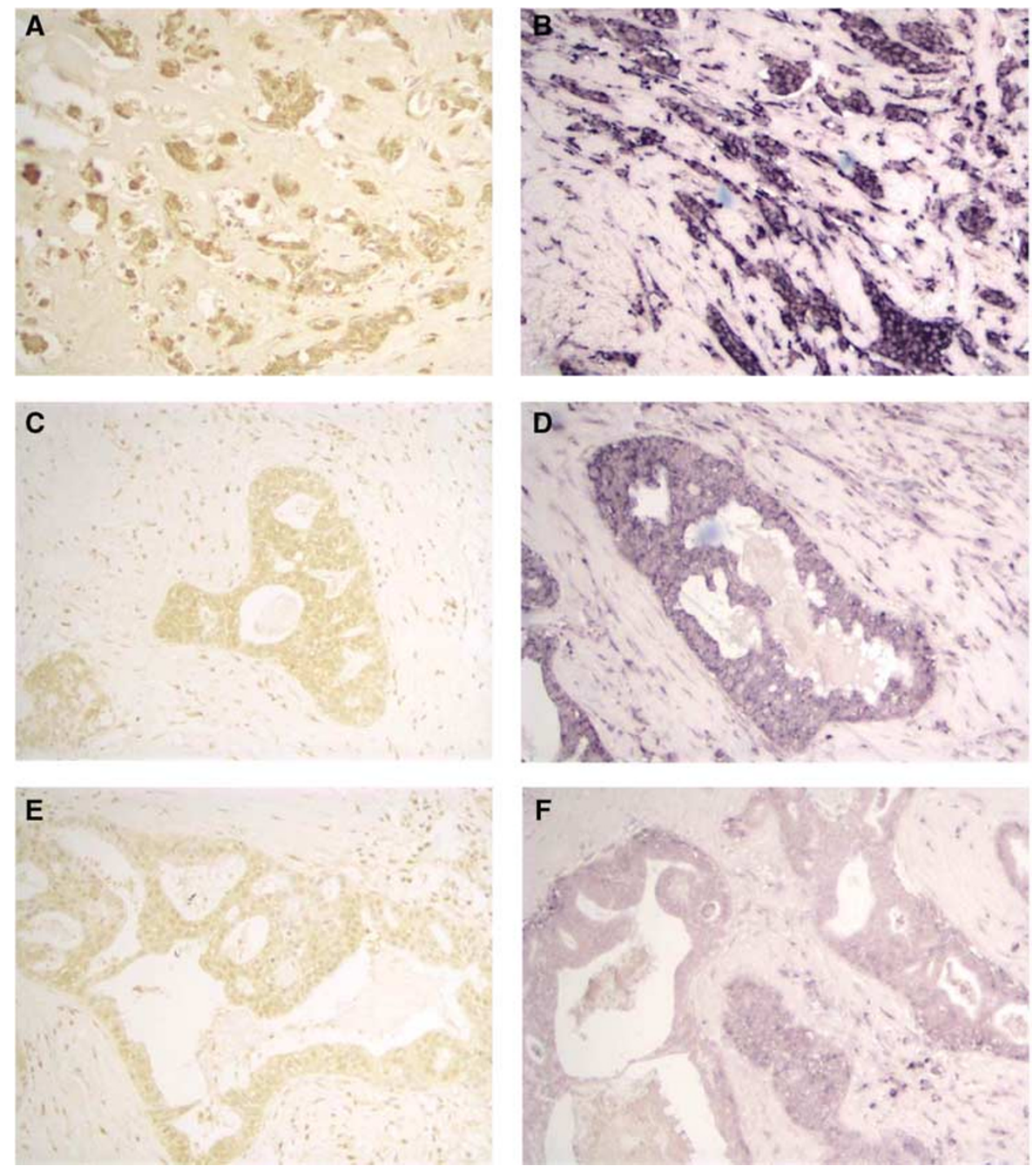

Figure 2 Immunohistochemical staining and in situ hybridisation for c-Myc of three sets of invasive ductal carcinoma. (A, C and $\mathbf{E})$ High ( $3+$ ), intermediate $(2+)$ and low $(I+)$ level of staining by immunohistochemistry for c-Myc. $(\mathbf{B}, \mathbf{D}$ and $\mathbf{F})$ High $(3+)$, intermediate $(2+)$ and low $(I+)$ level of staining by in situ hybridisation.

\section{DISCUSSION}

Although there have been many reports on c-myc amplification in human breast cancer (Liao and Dickson, 2000), there are only two published studies involving application of the FISH technique to unfixed, frozen sections (Persons et al, 1997; Visscher et al, 1997), and one prior study using FISH on an archival human tissue microarray (Schraml et al, 1999). Another recent study applied FISH to evaluate c-myc amplification in ductal carcinoma in situ
(DCIS) (Aulmann et al, 2002). Using the FISH technique on formalin-fixed, paraffin-embedded sections, we now show that $70 \%$ of high-grade breast cancer samples bear c-myc gene copy amplifications. Interestingly, the above-mentioned study, using FISH and focusing on DCIS, detected amplification of c-myc in only $20 \%$ of cases, but found a correlation of c-myc with increased tumour size and proliferation (Aulmann et al, 2002).

The level of amplification of c-myc in our study ranged between one and four additional copies of the gene; the majority (84\%) of 
the cases with the gene amplification gained only one to two copies, also consistent with FISH data reported for c-myc copy amplification in human metastatic prostate carcinoma tissues (Jenkins et al, 1997). The relationship between the level of c-myc gene copy amplification and the level its increased mRNA expression has been examined previously in breast cancer cell lines (Hyman et al, 2002). In general, it has been concluded that the two scores coordinate for $c-m y c$, as is the case for many breast cancer genes. However, only $44 \%$ of the highly amplified genes, in general, showed increased RNA expression, and only $10.5 \%$ of the highly overexpressed genes were gene copy-amplified in the cell line study (Hyman et al, 2002). Another analysis was conducted to study of relationships between gene amplification and expression of 6095 genes in 37 intermediate grade human breast tumours. This study demonstrated that $62 \%$ of the highly amplified genes also showed elevated expression; overall, a two-fold change in DNA copy number was associated with a 1.5 -fold change in mRNA levels. Overall, $12 \%$ of the variation in gene expression in the breast tumours studied was associated with gene copy number variation (Pollack et al, 2002). Further study of additional human breast tumours, at precisely defined grades and stages, will be necessary in order to more fully define the relationships between DNA copy numbers and expression of genes. The studies we report here indicate higher levels of c-Myc gene amplification and expression, than other previous reports in breast cancer. We believe that this is probably the result of our analysis of individual tumour cells in a well-defined set of high-grade breast tumours. Prior c-Myc expression and amplification microarray studies used tumour specimens which contain normal stromal components,

Table 3 Correlations between c-myc gene copy number (FISH) mRNA expression (ISH)

\begin{tabular}{l}
\hline \multicolumn{2}{c}{ Low } \\
\cline { 2 - 3 } (A) ISH (\% cells) \\
Low \\
High
\end{tabular}

potentially underestimating amplification and expression levels of the invasive tumour components (Pollack et al, 2002).

Our study reports a percentage of tumours gene amplified for c$m y c$ (using FISH in high-grade tumours) that is much higher than the average figure $(15.5 \%)$ reported in the literature (Isola et al, 2002). Most of the prior studies have employed the relatively insensitive Southern blot technique, and were reviewed in a recent meta-analysis (Deming et al, 2000). Consistent with this prior literature background, a recent study of 94 lobular and ductal breast cancers assessed amplification of c-myc by using a semiquantitative PCR assay and protein expression, with

Table 5 Nuclear/cytoplasmic localisation of c-Myc comparing normal and invasive cells

\begin{tabular}{lccc}
\hline $\begin{array}{l}\text { Normal cells } \\
\text { (frequency percent) }\end{array}$ & $\begin{array}{l}\text { Invasive cells } \\
\text { (frequency percent) }\end{array}$ & Tota \\
\hline (A) Nuclear localisation & & & \\
& - & 4 & \\
- & 28 & 8 & 32 \\
+ & 14 & 12 & 22 \\
Total & 42 & & 54
\end{tabular}

(B) Cytoplasmic localisation

\begin{tabular}{lrrr}
- & 12 & 13 & 25 \\
+ & 9 & 20 & 21 \\
Total & 21 & 33 & 54 \\
\hline
\end{tabular}

In all, 54 pairs (normal vs invasive) of tissues were analysed to answer the questions of (I) whether positivity of nuclear cells in normal tissues is different from that in invasive cells, and similarly (2) whether positivity of cytoplasmic cells in normal tissues is different from that in invasive cells. The data are summarised in the above contingency tables. In all, 22 normal cell specimens were positive for c-Myc staining (40.71\%), compared to 12 specimens (22.2\%) in invasive cells. The difference is statistically significant $(P=0.01)$ by McNemar's $\chi^{2}$ test (two-sided).

Table 6 Correlation between c-Myc protein expression (IHC) and cmyc gene copy number (FISH)

\begin{tabular}{lcc}
\hline & \multicolumn{2}{c}{ FISH } \\
\cline { 2 - 3 } IHC (\% cells) & Low & High \\
\hline & & \\
Low & 3 & 0 \\
High & 10 & 15 \\
& & $P=0.0016$
\end{tabular}

Consecutive serial sections of high-grade human breast tumours were scored for cmyc gene copy number or protein expression, by immunohistochemistry (IHC). $I H C$ scores were defined in the Materials and methods section. Data were analysed for correlations between the results. A highly significant correlation was observed between high c-Myc protein expression $(\mathrm{IHC})$ between percent cells positive and high c-myc gene amplification (FISH). $P=0.0016$ from two-sided McNemar's test. Note that for 15 cases, no staining for c-Myc could be detected; these negative cases were not included in the correlation presented, above.

Table 4 c-Myc immunohistochemistry (IHC) results

\begin{tabular}{|c|c|c|c|c|c|c|c|c|c|}
\hline Staining intensity & 0 & I & $\mathbf{2}$ & 3 & Percent positivity & $\mathbf{I}$ & 2 & 3 & 4 \\
\hline Number of tumour samples in each category & 15 & 13 & 20 & 3 & Number of tumour samples in each category & 2 & 2 & I & 29 \\
\hline
\end{tabular}

In all, 5 I high-grade human breast carcinomas were analysed to determine the relationships between c-Myc protein expression and c-myc gene in situ hybridisation results. Data are shown in two ways in the above table. First, overall staining intensity of c-Myc-positive cells was scored as 0, 1, 2, 3 (low to high), and the number of tumour samples at each level of staining is indicated on the line below. Next, in a random subset of these cases, the percentage of tumour cells staining was scored as 0, I, 2, 3, 4 (low to high \%, as discussed in Materials and methods). The number of tumours at each level of percent cell positivity for c-Myc is indicated on the line below. 
densitometry, after Western blot. These data showed c-myc gene amplification in $21 \%$ of tumours (Jenkins et al, 1997), using assays not based on in situ discrimination of tumour $v s$ nontumour cells. The lower frequency of $c-m y c$ in this prior study is in contrast with the data we present here, and could be the result of the higher sensitivity and precision of the FISH and immunohistochemical methods, as distinct from quantitative PCR and Western blot densitometry. In addition, the $70 \%$ of amplified tumours in our study is also much higher than the $12 \%$ reported by Schraml et al (1999), using a c-myc FISH test on a tissue microarray. This large difference may be because the arrays are prepared from cores of paraffin-embedded tissue, as small as $0.6 \mathrm{~mm}$ in diameter which may contain too few tumour cells for complete analysis of amplification of a gene, such as c-myc. c-myc is known to be quite heterogeneous in its gene amplification within individual tumours (in contrast to HER2/neu, for example) (Persons et al, 1997).

Most previous reports on the expression of c-myc mRNA have utilised Northern blot, dot blot or PCR-based approaches, while just a few involved in situ hybridisation, which were primarily performed on frozen tissue sections (Liao and Dickson, 2000). Normal breast tissue is dominated by adipose cells, differing greatly from tumour tissue in its epithelial cellularity. Thus, normal and tumour tissues may not be rigorously compared by techniques involving RNA extraction from total tissue. Therefore, conclusions such as 'increased expression' may be more difficult to make from studies with Northern blot, dot blot and PCR-based techniques that require RNA extraction from tissues that have not been fastidiously micro-dissected for selection of tumour cells. Using a more sensitive, nonradioactive in situ hybridisation (ISH) approach on formalin-fixed, paraffin-embedded sections, we report herein high expression of c-myc mRNA in $92 \%$ of highgrade breast carcinomas. This figure is much higher than the recently reported data (22\%), obtained by using a real-time RT PCR method (Bieche et al, 1999). Dilution of the RNA from epithelium by the RNA from adipose in normal breast tissue in this latest prior report may be one of the possible explanations for this large difference.

In conclusion, the present study shows that approximately 70,92 and $70 \%$ of biopsies of untreated high-grade breast cancer exhibit c-myc gene amplification, mRNA overexpression and protein overexpression, respectively. In most cases (84\%), with gene copy amplification, the c- $m y c$ gene gains one to two additional copies. c$m y c$ gene amplification was significantly associated with expression of its mRNA (both by intensity in invasive cells and by percentage positivity in invasive cells), and with expression of its protein (by percentage positivity in invasive cells). However, our data were also consistent with the prior literature on c-Myc (reviewed in Nass and Dickson, 1997; Liao and Dickson, 2000), indicating complex transcriptional, post transcriptional, translational and post-translational control of c-Myc expression in vitro. Specifically, in Table 5 we observed that in $40 \%$ of the high-grade tumours tested, c-Myc protein was expressed at high levels, despite a lack of its gene amplification.

It will be interesting to analyse lower grade tumours and premalignant lesions, with the same measurement tools, to determine if this $c-m y c$ amplification pattern is different, comparing different steps in onset and progression of the disease. Specifically, prior studies in fibroblasts and in human mammary epithelial cells (Liao et al, 1998, 2000a, b) have demonstrated that only a subtle deregulation of expression of cMyc is sufficient to allow genomic instability. These prior cell biologic findings raise the question of whether c-Myc protein expression precedes or follows its gene amplification during the course of the natural history of breast cancer. It will also be interesting for future studies of lower grade breast cancers and premalignant lesions to determine whether there is evidence of nuclear exclusion of $\mathrm{c}-\mathrm{Myc}$ protein. Indeed, nuclear exclusion of c-Myc in high-grade tumours could serve to attenuate its functions in later stages of disease progression (Liao and Dickson, 2000).

\section{ACKNOWLEDGEMENTS}

We would like to express our gratitude to Mr G Veytsman and $\mathrm{H}-\mathrm{K}$ Cao for scoring FISH data, Mrs S Constable for helping in tissue sectioning. This work was supported by NIH Grants RO1 CA72460 and AG1496 to RB Dickson and by a pilot grant from the Georgetown University ACS Institutional Research Grant to JK Blancato. This work was supported by NIH Grants RO1 CA72460 and AG1496 to RB Dickson and by an ACS pilot grant (from the GU Institutional ACS Research Grant) to JK Blancato.

\section{REFERENCES}

Augenlich L, Wadler S, Gorner G, Richards C, Ryan L, Multani A, Pathak S, Benson A, Haller D, Heerdt B (1997) Low-level c-myc amplification in human colonic carcinoma cell lines and tumors: a frequent, p53independent mutation associated with improved outcome in a randomized multi-institutional trial. Cancer Res 57: 1769-1775

Aulmann S, Bentz M, Sinn HP (2002) C-myc oncogene amplification in ductal carcinoma in situ of the breast. Breast Cancer Res Treat 74: $25-31$

Berns E, Klijn J, Van Putten W, van Staveren I, Portengen H, Foekens J (1992) c-myc amplification is a better prognostic factor than HER2/neu amplification in primary breast cancer. Cancer Res 52: 1107-1113

Bieche I, Laurendeau I, Tozlu S, Olivi M, Vidaud D, Lidereau R, Vidaud M (1999) Quantitation of MYC gene expression in sporadic breast tumors with a real-time transcription-PCR assay. Cancer Res 59: 2759-2765

Chana J, Cree I, Foss A, Hungerford J, Wilson G (1998a) The prognositc significance of $c-m y c$ oncogene expression in uveal melanoma. Melanoma Res 8: 139-144

Chana J, Grover R, Wilson G, Hudson D, Forders M, Sanders R, Grobbelaar A (1998b) The clinical significance of c-myc oncogene expression in melanomas of the scalp. Br J Plast Surg 51: $191-194$

Chana J, Wilson G, Cree I, Alexander R, Myatt N, Neale M, Foss A, Hungerford J (1999) c-myc, p53, and bcl-2 expression and clinical outcome in uveal melanoma. Br J Opthamol 83: 110-114

Deming S, Nass S, Dickson R, Trock B (2000) c-myc amplification in breast cancer: a meta-analysis of its occurrence and prognostic relevance. $\mathrm{Br} J$ Cancer 83: $1688-1695$

Diebold J, Suchy B, Baretton G, Blasenbreu S, Meier W, Schmidt M, Rabes H, Lohrs U (1996) DNA ploidy and MYC DNA amplification in ovarian carcinomas: correlation with p53 and bcl-2 expression, proliferative activity and prognosis. Virchows Arch 429: 221 - 227

Grover R, Chana J, Grobbelaar A, Hudson D, Forders M, Wilson G, Sanders $\mathrm{R}$ (1999) Measurement of $\mathrm{c}-m y c$ oncogene expression provides an accurate prognostic marker for acral lentiginous melanoma. Br J Plast Surg 52: $122-126$

Grover R, Ross D, Wilson G, Sanders R (1997) Measurement of c-myc oncoprotein provides an independent prognostic marker for regional metastatic melanoma. Br J Plast Surg 50: 478-482

Hyman E, Kauraniemi P, Hautaniemi S, Wolf M, Mousses S, Rozenblum E, Ringner M, Sauter G, Monni O, Elkahloun A, Kallioniemi O, Kallioniemi A (2002) Impact of DNA amplification on gene expression patterns in breast cancer. Cancer Res 62: 6240-6245

Isola J, Chu L, De Vries S, Matsumura K, Chew K, Ljung B, Waldman F (1999) Genetic alterations in ERBB2-amplified breast carcinomas. Clin Cancer Res 5: $4140-4145$

Jenkins R, Qian J, Lieber M, Bostwick D (1997) Detection of c-myc oncogene amplification and chromosomal anomalies in metastatic 
prostatic carcinoma by fluorescence in situ hybridization. Cancer Res 57: $524-531$

Liao D, Dickson R (2000) c-Myc in breast cancer. Endocrine-Relat Cancer 7: $143-164$

Liao D, Hou X, Bai S, Li S, Li J (2000a) Unusual deregulation of cell cycle components in early and frank estrogen-induced renal neoplasias in the Syrian hamster. Carcinogenesis 21: 2167-2173

Liao D, Natarajan G, Deming S, Jamerson M, Johnson M, Chepko G, Dickson R (2000b) Cell cycle basis for the onset and progression of cMyc-induced, TGFa-enhanced mouse mammary gland carcinogenesis. Oncogene 19: 1307 - 1317

Liao D, Pantazis C, Hou X, Li S (1998) Promotion of estrogen-induced mammary gland carcinogenesis by androgen in the male Noble rat: probable mediation by steroid receptors. Carcinogenesis 19: 2173-2180

Marcu K, Bossone S, Patel A (1992) Myc function and regulation. Annu Rev Biochem 61: $809-860$

Melhem M, Meisler A, Finley G, Bryce W, Jones M, Tribby I, Pipas J, Koski R (1992) Distribution of cells expressing Myc proteins in human colorectal epithelium, polyps, and malignant tumors. Cancer Res 52: $5853-5864$

Nass S, Dickson R (1997) Defining a role for c-Myc in breast tumorigenesis. Breast Cancer Res Treat 44: 1-22

Nesbit C, Tersak J, Prochownik E (1999) MYC oncogenes and human neoplastic disease. Oncogene 18: 3004-3016

Persons D, Borelli K, Hsu P (1997) Quantitation of HER-2/neu and c-myc gene amplification in breast carcinoma using fluorescence in situ hybridization. Mod Pathol 10: 720-727

Pietilainen T, Lipponen P, Aaltomaa S, Eskelinen M, Kosma V, Syrjanen K (1995) Expression of $\mathrm{c}-m y c$ proteins in breast cancer as related to established prognostic factors and survival. Anticancer Res 15: 959-964

Polacarz S, Hey N, Stephenson T, Hill A (1989) c-myc oncogene product p62 $2^{\text {c-myc }}$ in ovarian mucinous neoplasms: immunohistochemical study correlated with malignancy. J Clin Pathol 42: $148-152$

Pollack JR, Sorlie T, Perou CM, Rees CA, Jeffrey SS, Lonning PE, Tibshirani R, Botstein D, Borresen-Dale AL, Brown PO (2002) Microarray analysis reveals a major direct role of DNA copy number alteration in the transcriptional program of human breast tumors. Proc Natl Acad Sci USA 99: $12963-12968$

Sartelet S, Grossi L, Pasquier D, Conbaret V, Bouvier R, Ranchere D, Plantaz D, Munzer M, Philip T, Birembaut P, Zahm J, Bergeron C, Gaillard D, Pasquier B (2002) Detection of N-myc amplification by FISH in immature areas of fixed neuroblastomas: more efficient than Southern blot/PCR. J Pathol 198: 83-91
Sasano H, Nagura H, Silverberg S (1992) Immunolocalization of c-myc oncoprotein in mucinous and serous adenocarcinomas of the ovary. Hum Pathol 23: $491-495$

Sato H, Tsuchiya A, Abe R (1995) Correlation between c-myc protein expression and phases of the cell cycle in human colorectal carcinomas. Fukushima J Med Sci 41: $111-113$

Schraml P, Kononen J, Bubendorf L, Moch H, Bissig H, Noctito A, Mihatsch M, Kallioniemi O, Sauter G (1999) Tissue microarrays for gene amplification surveys in many different tumor types. Clin Cancer Res 5: $1966-1975$

Sikora K, Chan S, Evan G, Gabra H, Markham N, Stewart J, Watson J (1987) c-myc oncogene expression in colorectal cancer. Cancer 59: $1289-1295$

Sikora K, Evan G, Stewart J, Watson J (1985) Detection of the c-myc oncogene product in testicular cancer. Br J Cancer 52: 171-176

Smith D, Goh H-S (1996) Overexpression of c-myc proto-oncogene in colorectal carcinoma is associated with a reduced mortality that is abrogated by point mutation of the p53 tumor suppressor gene. Clin Cancer Res 2: 1049-1053

Sundaresan V, Forgacs I, Wight D, Wilson B, Evan G, Watson J (1987) Abnormal distribution of c-myc oncogene product in familial adenomatous polyposis. J Clin Pathol 40: 1274-1281

Visca P, Alo P, Del Nonno F, Bott C, Trombetta G, Marandino F, Filippi S, Di Tondo U, Donnorso R (1999) Immunohistochemical expression of fatty acid synthase, apoptotic-regulating genes, proliferating factors, and ras, protein product in colorectal adenomas, carcinomas, and adjacent nonneoplastic mucosa. Clin Cancer Res 5: 4111-4118

Visscher D, Wallis T, Awussah S, Mohamed A, Crissman J (1997) Evaluation of MYC and chromosome 8 copy number in breast carcinoma by interphase cytogenetics. Genes, Chromosomes, Cancer 6: $389-404$

Voravud N, Foster C, Gilbertson J, Sikora K, Waxman J (1989) Oncogene expression in cholangiocarcinoma and in normal hepatic development. Hum Pathol 20: 1163-1168

Watson J, Stewart J, Evan G, Ritson A, Sikora K (1986) The clinical significance of low cytometric c-myc oncoprotein in testicular cancer. $\mathrm{Br}$ I Cancer 53: 331 - 337

Williams A, Piris J, Wyllie A (1990) Immunohistochemical demonstration of altered intracellular localization of the $c-m y c$ oncogene product in human colorectal neoplasms. J Pathol 160: 287-293

Yuen M, Wu P, Lau J, Lai C (2001) Expression of c-Myc, c-Fos, and c-Jun in hepatocellular carcinoma. Cancer 91: $106-112$

Zar JH (1974) Biostatistical Analysis. Prentice-Hall: Englewood Cliffs, NJ 